\title{
Redox Properties of Cobalt Tetraphenyl Porphyrin Adsorbed on Semi-Conductor Oxides Studied by Photoacoustic Spectroscopy
}

\author{
A. M. MERLE, L. BRZEZINSKI, $\uparrow$ A. CHERQAOUI, C. GIANNOTTI
}

ERA 167 Photophysique et Photochimie moléculaire Université de Bordeaux 1, F33405 Talence Cedex France. †ENSCPB, Université de Bordeaux I. $¥ I C S N$, F91190, Gif-sur-Yvette

Photoacoustic spectroscopy has allowed to show that physisorbed $\mathrm{Co}^{\mathrm{II}} \mathrm{TPP}$ is ground state monooxidized into $\left(\mathrm{Co}^{\mathrm{III}} \mathrm{TPP}\right)^{+}$when adsorbed on $\mathrm{Nb}_{2} \mathrm{O}_{5}, \mathrm{TiO}_{2}, \mathrm{SrTiO}_{3}, \mathrm{ZnO}$, $\mathrm{SnO}_{2}$. A double oxidation is observed when the porphyrin is adsorbed on $\mathrm{WO}_{3}$. On the contrary when it is adsorbed on $\mathrm{ZrO}_{2}$ and $\mathrm{SiO}_{2}$, oxidation only occurs if the porphyrin has been driven by irradiation in its excited state. It remains in the reduced form when adsorbed on $\mathrm{Ta}_{2} \mathrm{O}_{5}$. This behavior has been correlated with the band positions of these various oxides and has allowed to roughly position the oxidation potential of CoTPP in adsorbed state $\sim 0.8 \mathrm{~V}$ higher than in solution $(0.5 \mathrm{~V} / \mathrm{SCE}$ or $5.3 \mathrm{~V} /$ vacuum) that is $4.5 \mathrm{~V} /$ vacuum.

\section{INTRODUCTION}

Photocatalytic effects of semi conductors have been under investigation for a long time because of their potential application to direct conversion of solar energy into chemical energy. ${ }^{1}$ The irradiation of the semi conductor with an energy higher than the band-gap is accompanied by the promotion of an electron from the valence band to the conduction band. Either the hole left in the valence band or the electron may be used for further chemical reaction ${ }^{2}$ or current generation. ${ }^{3}$ One of the problems of the photo electrochemical cells for solar energy storage is the choice of the semi conductor. When narrow band gap semi conductors which are good absorbers of solar 
spectrum are used such as CdS, a strong anodic decomposition is observed which degrades the semi conductor. On the other hand, the inconvenience of the wide band gap semi conductors such as $\mathrm{TiO}_{2}$, $\mathrm{SrTiO}_{3}$ which are stable against photo anodic decomposition is that they are poor absorbers of the solar spectrum. In order to remedy this situation it has been tempted to sensitize the semi conductor. The sensitization may be realized by introduction of impurity dopants in the semi conductor lattice such as $\mathrm{Cr}^{3+}$ ions in titanium dioxide ${ }^{4,5}$ or by chemical derivatization of the surface of the semi conductor. ${ }^{6}$ Another possibility consists in the adsorption of visible light absorbing organic material at the surface of the semi conductor. Most of the compounds which have been used in such an aim are dyes. ${ }^{7}$ It has been shown that they are oxidized and inject an electron into the semi conductor after being excited by absorption of light. ${ }^{8}$ The dynamics of such sensitization process has been studied in the case of rose bengal on $\mathrm{ZnO} .{ }^{9}$ Further studies have made evident that the quantum efficiency for the photoinjection of electrons, in the case of rose bengal on $\mathrm{TiO}_{2}$ was independent of the $\mathrm{pH}$ and of the dye surface concentration. $^{10}$

Porphyrins are other organic compounds which are good candidates for sensitization of semi conductors. They strongly absorb the visible light and are able to undergo a wide variety of electron exchanges. ${ }^{11}$ They have been tentatively used directly for water photooxidation ${ }^{12}$ and for photoinduced redox reactions in aqueous micelles. ${ }^{13}$ Their behavior when being adsorbed on electrodes has also been studied. When being deposited on a photo inactive electrode such as platinium or carbon, they may induce oxidation properties of the electrode that normally undergoes reduction when being illuminated. In the case of an aluminum electrode it has been shown that the system is photoactive and best described by a metal-insulator-semi conductor Schottky barrier consisting of $\mathrm{Al} / \mathrm{Al}_{2} \mathrm{O}_{3}$ /porphyrin where the porphyrin behaves as a $p$-type semi conductor. ${ }^{14}$ The sensitization of semi conductor electrodes by porphyrins has also been investigated. ${ }^{15,11}$ Photochemical measurements have shown that a $n$-type $\mathrm{SnO}_{2}$ semi conductor electrode acts as a photo cathode when sensitized by a layer of meso-tetraphenyl porphyrin ${ }^{16}$ instead of the usual photo anodic behavior.

However, it is to be noted that the interface porphyrin/electrode has never been studied directly as mostly electrochemical techniques have been used to investigate the sensitized electrode. One of the 
reasons for such a lack is the difficulty in applying classical spectroscopic techniques to the investigation of an opaque interface. Photoacoustic spectroscopy is a technique which has been recently developed that can overcome this problem as it allows the direct observation of the absorption spectrum of opaque samples. ${ }^{17}$ The sample, contained in a tight cell is irradiated with monochromatic modulated light. The absorbed energy is converted into a heat wave via the radiationless transitions. This heat wave propagates through the sample and the cell gas and transforms into a pressure wave within the cell which is detected with a microphone. The theoretical treatment done by Rosencwaig and Gersho ${ }^{18}$ shows that the microphone signal always depends upon the thermal properties of the sample and, when not saturated depends also on the optical properties of the sample. ${ }^{19}$ Furthermore, by varying the frequency of modulation of the light it is possible to determine these optical properties at various depth of the sample which is quite favorable for the study of an interface ${ }^{20}$ The photoacoustic spectrum is recorded by measuring the microphone signal as a function of the irradiation wavelength. It is truly a radiationless excitation spectrum.

Photoacoustic spectroscopy has been recently applied to photo electrochemical investigations. The change in the absorption spectrum of a $\mathrm{ZnO}$ electrode upon electrolysis has been investigated. ${ }^{21}$ Sensitization studies have just started: dye oxidation has been observed at fixed wavelength as a function of the applied potential on an $\mathrm{SnO}_{2}$ electrode. ${ }^{22}$ Chemical modification of the dye as a function of irradiation has been studied in the case of $\mathrm{RhB}$ adsorbed on $\mathrm{ZnO}$ which undergoes deethylation.

In this work, we have applied photoacoustic spectroscopy to the investigation of the interface obtained by adsorption of cobalt tetraphenyl porphyrin on various semi conductor oxides and insulators. In order to study the electron exchanges at this interface we have determined the oxidation state of the adsorbed porphyrin by observing its photoacoustic spectrum depending on the substrate and irradiation.

\section{EXPERIMENTAL}

Commercial semi conductor oxides provided by Merck have been used. It has been checked that a nitric acid etching had no influence 
on the observations. Thus the oxides have been used without any chemical treatment. Any how, in order to eliminate water and oxygen effects they have been heaten at $130^{\circ} \mathrm{C}$ during 12 hours, degassed up to $10^{-5}$ torr and kept under dry nitrogen until used.

The porphyrins have been prepared according to the literature. ${ }^{23}$ They have been further purified by thin layer chromatography on silicic acid Merck 60 and stored in the dark in absence of oxygen.

The adsorption of the porphyrin on the oxides was realized by allowing a methylen chloride solution of porphyrin to contact the degassed oxide. A $10^{-4} \mathrm{M}$ solution concentration was chosen as being a good compromise for having a good signal to noise ratio in the spectra and weak aggregation effects. It was checked that 2 hours contact were sufficient to allow the adsorption equilibrium to be reached. The surnagent solution was then eliminated by filtration, the oxide was vacuum dried and finally transferred under nitrogen pressure in the photoacoustic cell. A $20 \mathrm{mg}$ sample is needed for making a photoacoustic spectrum. Anyhow, in order to facilitate the manipulation we have worked with $100 \mathrm{mg}$ oxide and $1 \mathrm{ml} 10^{-4} \mathrm{M}$ porphyrin solution. Such an experimental procedure has been adopted in order to prevent undesirable oxygen and water effects. Indeed we have observed extra oxidation of the CoTPP when being adsorbed on $\mathrm{Al}_{2} \mathrm{O}_{3}$ in presence of oxygen. We have also observed that the photoacoustic spectrum of CoTPP adsorbed on $\mathrm{ZnO}$ is broadened and red shifted of as much as $5 \mathrm{~nm}$ in presence of water. Anyhow we have not studied this effect quantitatively.

We have calculated the surface concentration of the porphyrin. We have measured the specific surface of the oxides using BET technique and determined the amount of adsorbed porphyrin by eluting with chloroform the sample and measuring the optical density of the elution. Under the assumption that the molecule lies flat on the oxide one obtains a surface concentration varying from $10^{-2}$ to $10^{-1}$ monolayer depending on the oxide, which tends to demonstrate that the porphyrin is adsorbed in monomeric form. Indeed we have observed a significant broadening of the spectra when the methylen chloride solution concentration is raised from $10^{-4} \mathrm{M}$ to $10^{-3}$ or $10^{-2} \mathrm{M}$ indicating in such a case the existence of aggregates.

The photoacoustic spectra have been taken in a EDT instrument. We have checked by measuring the frequency dependence of the signal $^{20}$ that there was no saturation at the frequency of $80 \mathrm{~Hz}$ which 
has been used for all the spectra. The experimental spectral resolution was $4 \mathrm{~nm}$ and it has been checked that it was not a limitation for the observations.

\section{RESULTS}

The photoacoustic spectra of the various oxides are represented on Figure 1. They are in agreement with the bands position determined by other methods. ${ }^{24,25,26}$

The spectral modifications of the porphyrin induced by adsorption are shown on Figure 2 in the case of the free base tetraphenyl porphyrin that undergoes less easy oxidation than CoTPP. The major features are the broadening of the spectrum and the decrease of the intensity ratio of the Soret band relative to the visible ones which is due to interaction with the substrate in the adsorbed state.

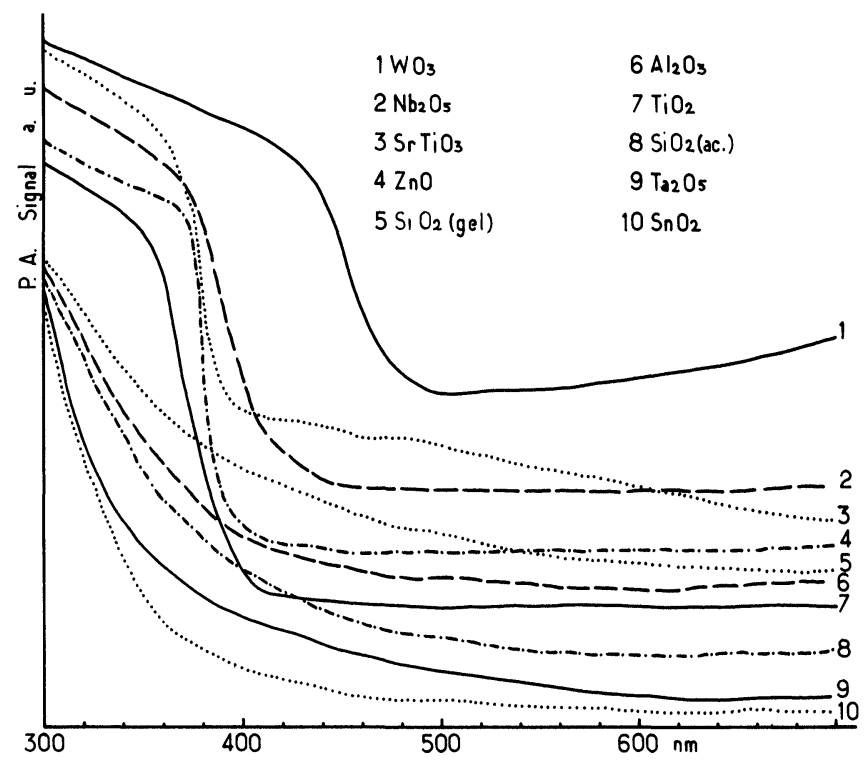

FIGURE 1 Photoacoustic spectrum of the degassed oxides (when water is present red shifts in the spectra may be observed). 


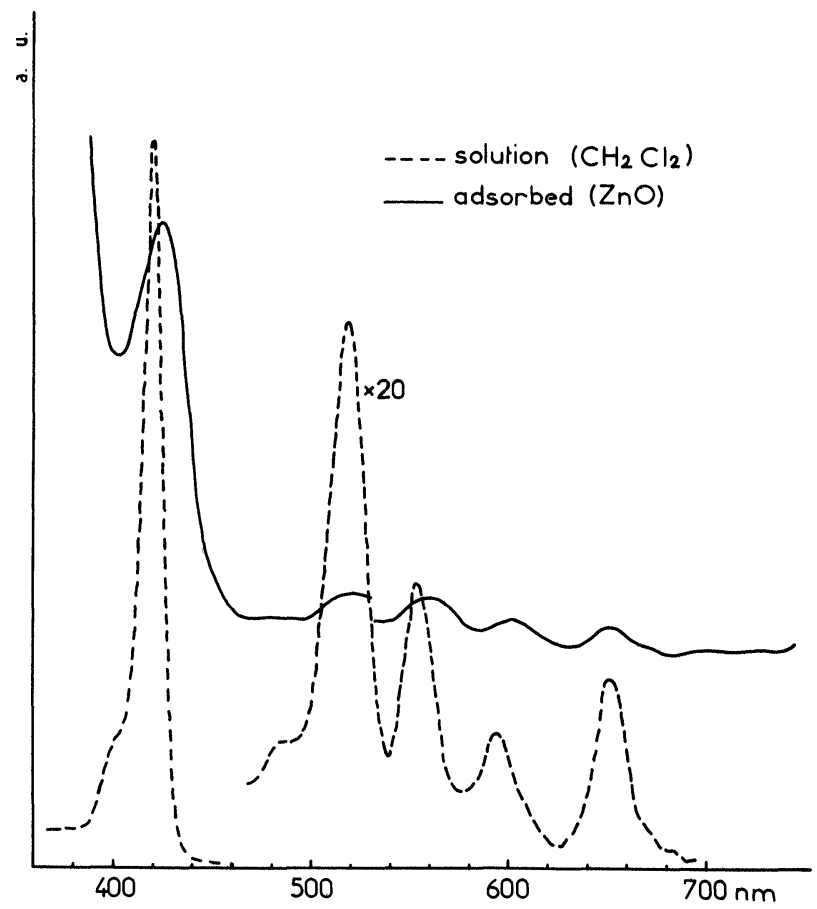

FIGURE 2 Comparison of the photoacoustic spectrum of tetraphenyl porphyrin in $\mathrm{CH}_{2} \mathrm{Cl}_{2}$ solution (-- ) and adsorbed on $\mathrm{ZnO}(-)$.

When cobalt porphyrin has been adsorbed on an oxide, the observed photoacoustic spectrum is the sum of the porphyrin and of the oxide absorption spectra because of the weak porphyrin surface concentration. It has been checked that an increase in the frequency modulation of the light which allows a more superficial observation of the sample ${ }^{20}$ did not eliminate the oxide absorption. When this latter interfered with the porphyrin absorption, deconvolution of the spectra has been done.

In solution, the valency of the cobalt atom in cobalt tetraphenyl porphyrin is II. The optical spectrum, of Co ${ }^{\text {II }}$ TPP consists in a Soret band at $415 \mathrm{~nm}$ and a broad visible band at $540 \mathrm{~nm} .{ }^{27}$ Easy oxidation may occur through valency change of the central metal atom. The $\left(\mathrm{Co}^{\mathrm{III}} \mathrm{TPP}\right)^{+}$has a red shifted optical spectrum with a Soret band at $438 \mathrm{~nm}$ and two visible bands at 550 and $590 \mathrm{~nm} .^{28,29}$ The second 
oxidation step takes place at the ligand and $\left(\mathrm{Co}^{\mathrm{III}} \mathrm{TPP}\right)^{++}$exhibits a weak absorption spectrum with a broad band around $600 \mathrm{~nm}^{28}$

In adsorbed state we have observed that the cobalt porphyrin has the same behavior as in solution: depending on the oxide the three types of spectra which have just been described may be observed which proves that oxidation takes place in the adsorbed state in the same way as it does in solution i.e., via the metal first and then via the ligand. The Co ${ }^{\mathrm{II}} \mathrm{TPP}$ spectrum is observed when the porphyrin is adsorbed on $\mathrm{Al}_{2} \mathrm{O}_{3}$ and $\mathrm{Ta}_{2} \mathrm{O}_{5}$. It consists in a Soret band at $400 \mathrm{~nm}$ and a visible band at $520 \mathrm{~nm}$ (Figure 3 ). We have observed that in both cases when the concentration of the porphyrin solution in contact with the oxide is lowered, the mono-oxidized form of the porphyrin appears $\left(10^{-5} \mathrm{M}\right)$ and becomes preponderant $\left(10^{-6} \mathrm{M}\right)$.

On $\mathrm{SiO}_{2}$ and $\mathrm{ZrO}_{2}$ substrates, the Co ${ }^{\mathrm{II}} \mathrm{TPP}$ spectrum is initially observed. However, photo-oxidation occurs as Co ${ }^{\text {II }}$ TPP transforms

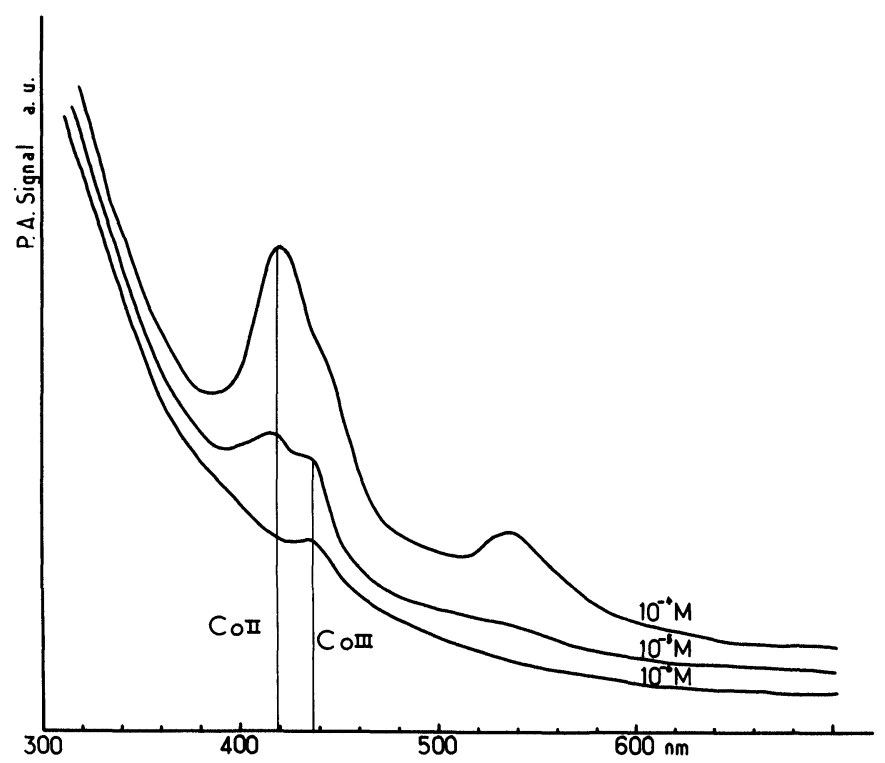

FIGURE 3 Photoacoustic spectrum of cobalt tetraphenyl porphyrin adsorbed on $\mathrm{Ta}_{2} \mathrm{O}_{5}, \mathrm{CO}^{\mathrm{II}} \mathrm{TPP}$ spectrum is preponderant at $10^{-4} \mathrm{M}$. As lowering the concentration to $10^{-6} \mathrm{M}$ the $\left(\mathrm{Co}^{\mathrm{III}} \mathrm{TPP}\right)^{+}$spectrum appears. The indicated concentration is the porphyrin $/ \mathrm{CH}_{2} \mathrm{Cl}_{2}$ solution one. 
in $\left(\mathrm{Co}^{\mathrm{III}} \mathrm{TPP}\right)^{+}$upon excitation of the porphyrin as made evident on Figure 4 . The irradiation has been done by $5 \mathrm{~s}$ steps with an XBO $450 \mathrm{~W}$ lamp filtered in such a way that only light at higher wavelength that $400 \mathrm{~nm}$ could pass.

When the porphyrin is adsorbed on all the other oxides except $\mathrm{WO}_{3}$ that is: $\mathrm{SnO}_{2}, \mathrm{Nb}_{2} \mathrm{O}_{5}, \mathrm{TiO}_{2}, \mathrm{ZnO}, \mathrm{SrTiO}_{3}$ both type spectra $\left(\mathrm{Co}^{\mathrm{II}} \mathrm{TPP}\right.$ and $\mathrm{Co}^{\mathrm{III}} \mathrm{TPP}^{+}$) are concomitantly observed (Figure 5). In all these cases photo-oxidation is not observed. However, at long irradiation time $(60 \mathrm{~s})$ a photodestruction of the cycle occurs as the spectrum vanishes without the apparition of new bands. This photochemistry is more efficient on the already oxidized form of the porphyrin.

In the case of $\mathrm{WO}_{3}$ the spectrum vanishes and, after subtracting the absorption of the oxide, one observes a broad and weak band that we have interpreted as due to the absorption of the doubly oxidized form $\left(\mathrm{Co}^{\mathrm{III}} \mathrm{TPP}\right)^{++}$.

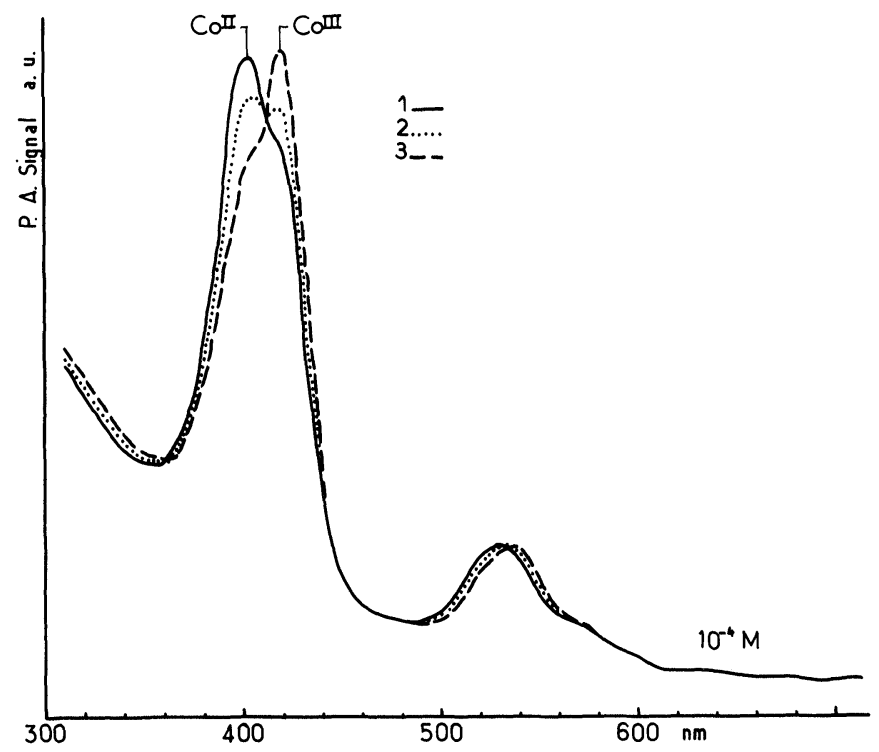

FIGURE 4 Photoacoustic spectrum of cobalt tetraphenyl porphyrin adsorbed on $\mathrm{ZrO}_{2}$. Evidence for photooxidation: (1) without irradiation (CO'I TPP), (2) $5 \mathrm{~s}$ irradiation, (3) $10 \mathrm{~s}$ irradiation $\left(\mathrm{Co}^{\mathrm{III}} \mathrm{TPP}\right)^{+}$. 


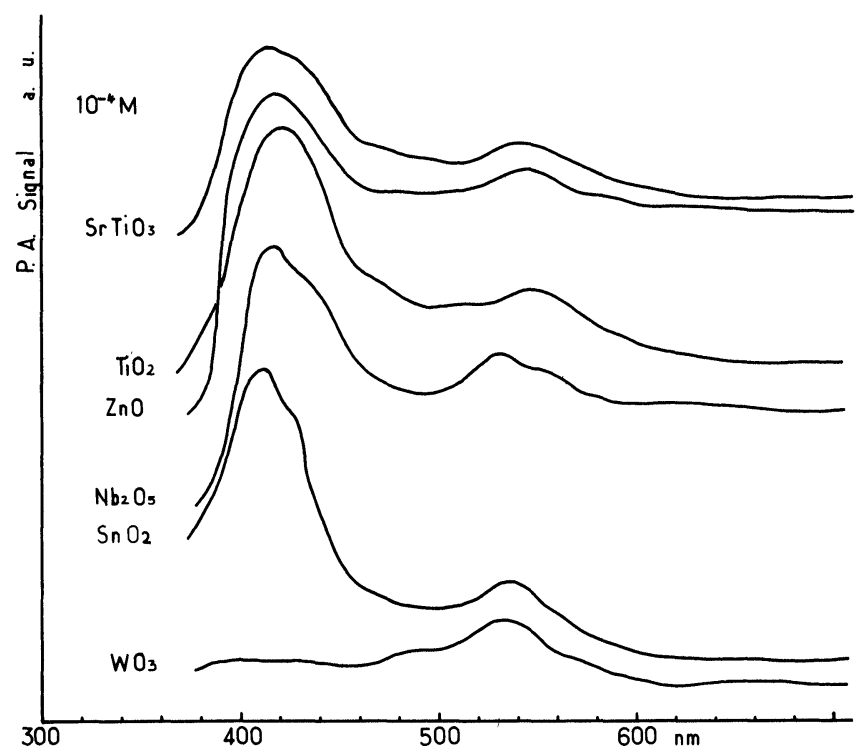

FIGURE 5 Photoacoustic spectra of cobalt tetraphynyl porphyrin adsorbed on $\mathrm{SnO}_{2}$, $\mathrm{Nb}_{2} \mathrm{O}_{5}, \mathrm{TiO}_{2}, \mathrm{ZnO}, \mathrm{SrTiO}_{3}$. The non oxidized and the monooxidized form are both present.

\section{DISCUSSION}

We have tempted to interprete the observations relating the energy levels of the semi conductors with the oxidation potential of the porphyrin. We shall reference them to vacuum level as all observations have been made in absence of electrolyte. A conversion to standard calomel electrode is done by substracting $4.75 \mathrm{~V}$ which is the potential of the electrode relative to vacuum.

The bands energies of the oxides are indicated on Figure 6. The positions of the Fermi levels have been obtained by the calculated electronegativities. ${ }^{24}$ The positions of the conduction bands are obtained by the electron affinities calculations or flatband potential measurements. ${ }^{24,25}$ The valence bands are positioned from the knowledge of the band gaps. ${ }^{25,26}$

The oxidation potentials of the cobalt porphyrin measured in $0.1 \mathrm{M}$ $n-\mathrm{Bu}_{4} \mathrm{NBF}_{4} /$ benzonitrile solution and corrected for electrolyte effect are indicated in Ref. 28. The metal oxidation potential is 


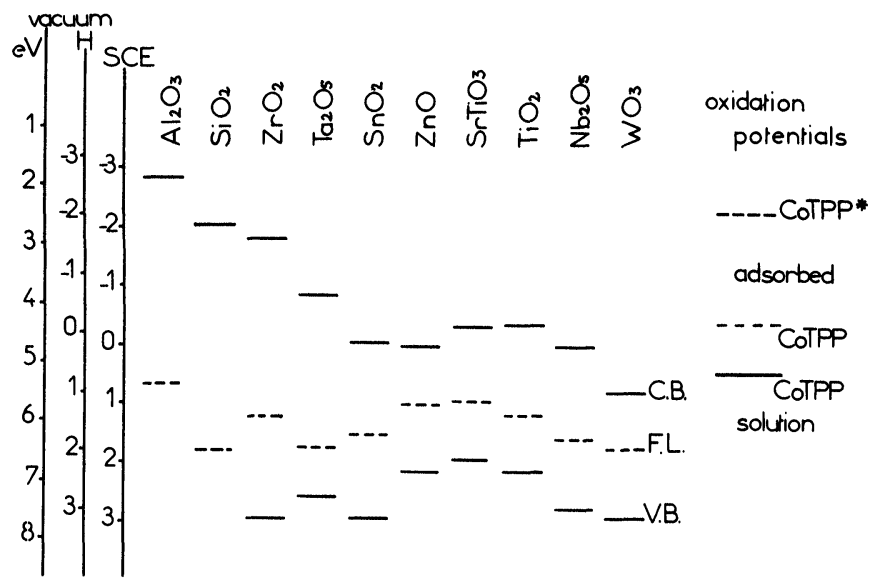

FIGURE 6 Energy diagram of the oxides and oxidation potentials of the porphyrin. The observations suggest that the oxidation in adsorbed state is higher than in $\mathrm{CH}_{2} \mathrm{Cl}_{2}$ solution.

$5.27 \mathrm{~V} /$ vacuum $(0.52 \mathrm{~V} / \mathrm{SCE})$ and the ligand second oxidation potential is at $5.94 \mathrm{~V} /$ vacuum $(1.19 \mathrm{~V} / \mathrm{SCE})$. One has to note that the monooxidation potential lies at lower energy than the conduction band of all the oxides (Figure 6). It is thus difficult to account for different oxidative behaviors when the porphyrin is deposited on the different oxides and furthermore to account for any oxidation of $\mathrm{Co}^{\mathrm{II}} \mathrm{TPP}$ which is usually interpreted as isoenergetic electron transfer from the porphyrin to the semi conductor conduction band. Thus we propose, in order to interprete the observations that the oxidation potential of tetraphenyl porphyrin in adsorbed state lies at higher energy than in solution.

We have tentatively positioned the oxidation potential of the porphyrin in the adsorbed state with the following hypotheses:

The porphyrin oxidation potential in adsorbed state does not depend on the oxide which is justified by the fact that we try to make only an evaluation of this potential.

The oxidation potential of the excited porphyrin differs from the ground state one only by the energy difference between the ground state and the first excited singlet state.

Ground state oxidation as well as excited state oxidation occur via an isoenergetic transfer from the porphyrin to the conduction band 
of the semi conductor at the energy of highest density of states in the conduction band that is about $0.8 \mathrm{eV}$ higher than the lower limit.

No band bending will be considered for the interpretation as the observations have been done in absence of electrolyte.

With these hypotheses and considering that CoTPP is photo oxidized when adsorbed on $\mathrm{SiO}_{2}$ and $\mathrm{ZrO}_{2}$, we may position the oxidation potential of excited CoTPP at the level of the conduction band of these oxides that is around $2.5 \mathrm{~V} /$ vacuum. Taking into account that the energy difference between the ground and the excited state of CoTPP is $2 \mathrm{eV}$ we may position the ground state oxidation potential of CoTPP at $4.5 \mathrm{eV} /$ vacuum which is about $0.8 \mathrm{eV}$ higher than the corresponding potential in solution. Such a value is quite reasonable as it is known that, in solution, changes in solvant polarity induce shifts in the oxidation potential up to $0.5 \mathrm{~V}$. Thus it is not surprising to find such an important change when going from solution to adsorbed state. The ground state oxidation potential evaluated in such a way lies at higher energy than the conduction level of $\mathrm{SnO}_{2}, \mathrm{Nb}_{2} \mathrm{O}_{5}$, $\mathrm{TiO}_{2}, \mathrm{ZnO}, \mathrm{SrTiO}_{3}$. It is thus understandable that oxidation may take place in the ground state when the porphyrin is adsorbed on those substrates. However it is not complete and is almost inexistant in the case of $\mathrm{Ta}_{2} \mathrm{O}_{5}$ as the conduction band of this latter lies at slightly higher energy (Figure 6).

It is possible to make an evaluation of the second ground state oxidation potential in the same way; one finds $5.1 \mathrm{~V} /$ vacuum, a value which is higher than the lowest level of the conduction band of $\mathrm{WO}_{3}$ which explains the double oxidation in this case (Figure 6).

The $\mathrm{Al}_{2} \mathrm{O}_{3}$ conduction band lies at such a high energy (Figure 6) that neither excited state oxidation nor (a fortiori) ground state oxidation of the porphyrin is possible when it is adsorbed on this substrate. The apparition of $\left(\mathrm{Co}^{\mathrm{III}} \mathrm{TPP}\right)^{+}$spectrum when lowering the concentration in the case of $\mathrm{Al}_{2} \mathrm{O}_{3}$ or $\mathrm{Ta}_{2} \mathrm{O}_{5}$ may be interpreted in term of imperfect degassing of the powder ${ }^{29}$ or presence of lattice impurities creating low lying states that allow oxidation of the porphyrin.

\section{CONCLUSION}

Photoacoustic spectroscopy has allowed to directly study the interface adsorbed porphyrin/semi conductor oxide by observation of the porphyrin spectrum. It has shown that: 
The redox properties of the cobalt tetraphenyl porphyrin are similar in solution and in adsorbed state.

The oxidation potential of the cobalt porphyrin in adsorbed state is higher than in solution.

However, this technique has only allowed to study the interface in absence of electrolyte. Further work is in progress using beam depletion techniques in order to study the oxidation state of the porphyrin in presence of an electrolyte that is in a more realistic condition for an application of the porphyrin sensitized semi conductor system for energy storage.

\section{References}

1. K. J. Zamaraev and V. N. Parmon, Catal. Rev. 22, 261 (1980).

2. M. A. Fox and C. C. Chen, J. Am. Chem. Soc. 103, 6757 (1981).

3. J. M. Mountz and H. Ti Tien, Solar Energy 21, 291 (1978).

4. G. Campet, M. P. Dare-Edwards, A. Hamnett and J. B. Goodenough, Nouveau Journal de Chimie 4 (8/9), 501 (1980).

5. E. Borgarello, J. Kiwi, M. Grätzel, E. Pelizzetti and M. Visca, J. Am. Chem. Soc. 104, 2996 (1982).

6. M. S. Wrighton, ACS Symposium Series $n^{\circ} 192$, Chemically Modified Surfaces in Catalysis and Electrocatalysis, ed. J. S. Miller (1982).

7. T. Iwasaki, S. Oda, H. Kamada and K. Honda, J. Phys. Chem. 84, 1060 (1980).

8. H. Gerischer, Photochem. Photobiol. 16, 243 (1972).

9. T. Kojima, T. Ban, K. Kasatani, M. Kawasaki and H. Sato, Chem. Phys. Lett. 91 (4), 319 (1982).

10. M. T. Spitler and M. Calvin, J. Chem. Phys. 66 (10), 4294 (1977).

11. I. Mochida, K. Tsuji, K. Suetsugu, H. Fujitsu, K. Takeshita,

12. A. Harriman, G. Porter and P. Walters, J. Photochem. 183 (1982).

13. R. H. Schmehl and D. G. Whitten, J. Phys. Chem. 85, 3473 (1981); D. Kessel and E. Rossi, Photochem. Photobiol. 35, 37 (1982).

14. K. Yamashita, N. Kihara, H. Shimidzu and H. Suzuki, Photochem. Photobiol. 35, 1 (1982).

15. J. M. Mounts and H. Ti-Tien, Solar Energy 21, 291 (1978).

16. H. Ti-Tien and J. Higgins, J. Electrochem. Soc. 127 (7), 1475 (1980).

17. M. J. Adams, B. C. Beadle, A. A. King and G. F. Kirkbright, Analyst 101, 553 (1976).

18. A. Rosencwaig and A. Gersho, J. Appl. Phys. 47 (1), 64 (1976).

19. J. F. McClelland and R. N. Kniseley, Appl. Phys. Lett. 28, 467 (1976).

20. H. S. Bennett and R. A. Forman, J. Appl. Phys. 48 (4), 1432 (1977).

21. H. Masuda, A. Fujishima and K. Honda, Bull. Chem. Soc. Jpn. 55, 672 (1982).

22. T. Iwasaki, T. Sawada, H. Kamada, A. Fujishima and K. Honda, J. Phys. Chem. 83 (16), 2142 (1979).

23. J. H. Fuhrhop and K. M. Smith, Laboratory Methods in Porphyrins and Metallo Porphyrins Research (Elsevier Scientific Publishing Company, Amsterdam, 1975).

24. M. A. Butler and D. S. Ginley, J. Electrochem. Soc. 25, 228 (1978). 
25. H. P. Maruska and A. K. Ghosh, Solar Energy 20, 443 (1978).

26. A. Smakula, Optica Acta 9, 206 (1962).

27. M. Gouterman, J. Mol. Spectr. 6, 138 (1961); A. Corwin, Ann. New York, Acad. Sci. 206, 201 (1973).

28. A. Wolberg and J. Manassen, J. Am. Chem. Soc. 92 (10), 2982 (1970).

29. P. Neta, J. Phys. Chem. 85, 3678 (1981). 